\title{
Recherche impliquant des êtres humains
}

Prof. Peter Suter, Genève

Président de l'ASSM

\section{Programme}

9h30 Allocution de bienvenue

Prof. Peter M. Suter, président de l'ASSM, Genève

9h40 Ein Gesetz über «Forschung am Menschen»: Was wollen die Behörden? Prof. Thomas Zeltner, directeur de l'Office fédéral de la santé publique, Berne

10h10 Kein medizinischer Fortschritt ohne Forschung am Menschen Prof. Peter Meier-Abt, vice-recteur «recherche», Université de Bâle

10h40 Forschung am Menschen: die sozialwissenschaftliche Perspektive Prof. Ulrike Ehlert, directrice de l'Institut psychologique de l'Université de Zurich

$11 \mathrm{~h} 10$ Pause café

11h30 Consentement chez le sujet de recherche incapable de discernement Prof. François Lemaire, Hôpital Henri-Mondor, Créteil (France)

12h00 The proposed law in Norway: critical perspectives on certain aspects of legal regulation of medical research Prof. Knut Ruyter, National Ethics Committee for Research, Oslo (Norway)

12h30 Repas 


\section{3h30 Ateliers}

I Tâches des commissions d'éthique

Experts: Giovan Maria Zanini, Bellinzona; avocat Michael Gerber, OFSP, Berne Modération: Prof. Dominique Sprumont, IDS, Neuchâtel

II Couverture des dommages

Experts: Dr Christoph Zenger, Berne; lic. iur. Jean-Christophe Méroz, Swissmedic, Berne Modération: Prof. Daniel Scheidegger, FNS, Bâle

III Sujets de recherche particulièrement vulnérables

Experts: Prof. Susanne Suter, Genève; lic. iur. Dolores Krapf, OFSP, Berne

Modération: Paul Dietschy, Swissmedic, Berne

IV Recherche en sciences sociales

Experts: Dr Alain Kaufmann, Lausanne; Dr Verena Schwander, OFSP, Berne Modération: Prof. Joseph Jurt, CSST, Freiburg (D)

$V$ Recherche sur du matériel biologique et des données personnelles (incl. biobanques)

Experts: Prof. Volker Dittmann, Bâle; Dr Andrea Arz de Falco, OFSP, Berne

Modération: Dr Margrit Leuthold, ASSM, Bâle

VI Recherche sur des personnes décédées

Experts: Prof. Robert Maurer, Zurich; Biol., Pol. Wiss. Katja Manike, OFSP, Berne Modération: Prof. Stephanie Clarke, ASSM, Lausanne

VII Recherche sur le tissu foetal

Experts: Prof. Wolfgang Holzgreve, Bâle; dipl. biol., lic. iur. Matthias Bürgin, OFSP, Berne Modération: Prof. Alex Mauron, CSST, Genève

15h00 Pause café

15h30 Rapports des ateliers

16h45 Panel: Limites de la réglementation juridique de la recherche impliquant des êtres humains

Participants: Prof. Daniel Scheidegger, Bâle; Prof. Peter Meier-Abt, Bâle; Prof. Ulrike Ehlert, Zurich; Dr Thomas Cueni, secrétaire général Interpharma, Bâle;

Prof. Dominique Sprumont, Institut de droit de la santé, Université de Neuchâtel

18 h00 Conclusions

Prof. Peter M. Suter, Genève

Inscription sur www.assm.ch. 